\title{
Structural Monitoring for the Rail Industry using Conventional Survey, Laser Scanning and Photogrammetry
}

\author{
Anita Soni $^{1}$, Stuart Robson ${ }^{1}$, Barry Gleeson ${ }^{2}$ \\ ${ }^{1}$ Dept. of Civil, Environmental \& Geomatic Engineering, University College London, Gower \\ Street, London, WC1E 6BT UK \\ ${ }^{2}$ Network Rail, James Forbes House, 27 Great Suffolk Street, London, SE1 0NS \\ Corresponding author: anita.soni.10@ucl.ac.uk
}

\begin{abstract}
Monitoring the movement of structures on railway projects in the UK typically involves the fixing of targets (e.g. prisms) or sensors onto the structures being monitored and their surroundings. Whilst this provides discrete point measurement capability across the structure, it is highly intrusive and expensive to setup in a railway environment.

Terrestrial Laser Scanning (TLS) has become an invaluable method of data capture within the surveying industry including applications such as deformation monitoring. The main advantages of TLS, as opposed to other surveying techniques, are the ability to capture large volumes of 3D data at high speed, remotely and with a reasonably high accuracy. A complimentary technique, Close Range Photogrammetry (CRP), has been traditionally applied to structural monitoring, but is not routinely considered by the railway monitoring community. This technique has the advantage of rapid data capture from a mobile camera with the capability to monitor single points or to generate a point cloud, with an equipment cost approximately twenty times cheaper than a TLS system and at about one tenth the cost of a single instrument total station approach.

This paper describes the application of TLS and CRP, along with conventional survey techniques, to the monitoring of a set of masonry arches during a major station refurbishment. Firstly, it investigates the capabilities of using TLS compared to traditional survey methods and encompasses a case where significant movements occur over an extended period of time. Interepoch comparison demonstrates a capability to detect change but highlights a requirement to understand the structure and data quality in making valid interpretations. Secondly the paper compares TLS and CRP techniques as monitoring tools for creating point cloud data on the same set of masonry arches. These investigations generate significant volumes of data conferring the additional challenge of how to visualise observed changes and communicate those changes and their significance to the engineers who must make informed decisions from the data in a timely fashion.
\end{abstract}




\section{Introduction}

\subsection{Project Background}

Network Rail is the owner and operator of the rail infrastructure in Great Britain. The Thameslink Programme (TLP) at Network Rail involves a $£ 6$ billion upgrade of the Thameslink railway network, which runs from Bedford to Brighton through Central London (Figure 1). This requires the redevelopment of major stations within the network over the project period which began in 2008 and is proposed to be completed by December 2018.

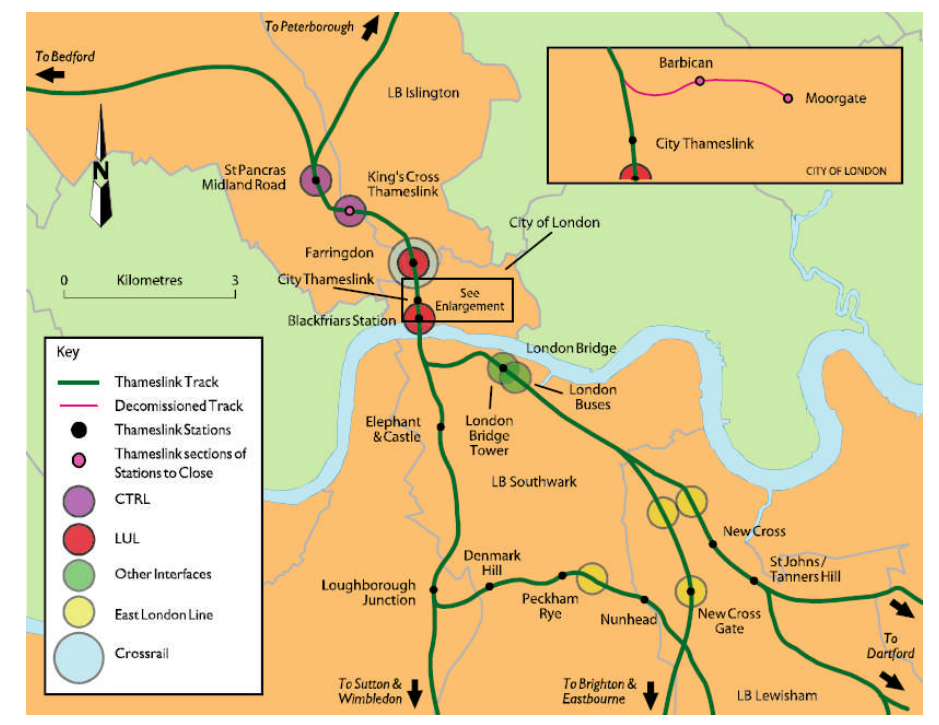

Figure 1 - Scope of Thameslink railway network running through Central London

As with any railway redevelopment programme, a key priority is to minimise disruption to retained assets belonging to Network Rail and its neighbours, including operational activities. Any works which have the potential to impact structural stability or asset performance require such impacts to be calculated and mitigated where possible. Therefore a monitoring strategy and specification is produced by the engineers which provides details of the predicted movement impacts along with the monitoring system to be implemented, including its accuracy and required frequency. An impact zone for different stages of the works is produced, as well as the identification of the assets that could potentially be affected. An alarm system is setup with trigger threshold values which depend on the structural stability or tolerance allowed for each feature. Monitoring is then implemented based on the strategy and specification documents.

\subsection{Monitoring practices in the railway industry}

Deformation monitoring through traditional surveying allows the measurement of reference and monitoring points. Whilst some systems incorporate inertial type sensors (tilt, crack sensors), the majority of traditional railway structural monitoring is based on the use of a Total Station (TS) observing to retro-reflecting glass prism targets. It's a well-known and established method for monitoring due to its high accuracy, precision and repeatability.

Despite this being the most conventional approach for structural monitoring, there are some inherent disadvantages with its implementation, particularly for the railway environment. Typically access to track or structures for installation requires significant notification periods which can be time consuming and costly. Once access has been approved, the instrumentation and targets must be fixed 
into position through drilling, clamping or gluing which is intrusive. When systems have been installed the system is prone to missing data due to occlude lines of sight between the instrument and target as works takes place. Targets are often knocked or destroyed and these interferences can set off "false movement" triggers which require an engineer to investigate further, potentially causing unnecessary delays and disruptions.

These types of limitation can lead to huge infrastructural costs whilst running a monitoring system. Typically for a major station redevelopment, monitoring alone can cost up to $£ 1$ million per year. Additionally through targeting, the monitoring system represents movement of the target that is attached to the structure rather than the movement of the structure itself. Therefore this does not represent true movement of the structure, each case having to be clarified for appropriate action to be taken.

With these huge costs and logistical limitations of a targeting monitoring setup, there is a need to investigate alternative approaches with the potential for a "target-less" monitoring measurement tool, for example through TLS and CRP.

\section{TLS and CRP for Deformation Monitoring}

\subsection{Terrestrial Laser Scanning}

TLS has become an invaluable method of data capture within the surveying industry. The main advantage of TLS, as opposed to traditional survey techniques, is the ability to remotely capture large volumes of 3D data at a high speed with reasonably high accuracy. Set against this is that the metric qualities of the 3D data are highly dependent on the reflectance and local geometry of the surfaces under observation and sample those surfaces in a regular grid dictated by angular instrument settings rather than the features being measured. As the technology has matured, manufacturers have produced scanners that have become faster with an increasing level of accuracy.

For the majority of survey projects, particularly on construction sites, the data is required to be delivered on a fixed co-ordinate system. For TLS this process is known as geo-referencing (Schuhmacher and Böhm, 2005). Traditionally this can be carried out by co-ordinating targets or points of interest using a TS (Allan, 2007; Lerma Garcia et al, 2008; Uren and Price, 2010) which gives millimetric accuracy, or by GPS (Schuhmacher and Böhm, 2005;Reshetyuk, 2010) which gives centimetre to metre levels of accuracy. Technology has also allowed scan geo-referencing via the inclusion of GPS within the scanner, for example a Reigl VZ-6000 (Riegl, 2014) and Faro X330 (FARO, 2014) system. However, due to the indoor environment, this type of solution was not suitable for this case study.

Case studies show how the benefits of TLS can be used as a monitoring/inspection tool for a wide variety of natural and man-made structures, for example: steel beams (Gordon et al, 2005; Park et al, 2007); rockfall events (Abellán et al, 2009 \& 2011; Alba and Scaioni, 2010); dams and locks (Lindenbergh and Pfeifer, 2005; Alba et al, 2006; Schneider, 2006); bridges and underpasses (Werner and Morris, 2010; Riveiro et al, 2011; Puente et al, 2012; Kopáčik, 2013); and tunnels (Nuttens et al, 2010, 2012, 2014) as well as more unique monitoring situations, for example Vezočnik (2009) which required a high pressure underground pipeline within a geologically unstable area to be monitored. One of the highlighted issues with using TLS, compared to high precision TS, is the limited precision of the single TLS points for making deformation measurements. This can be overcome by exploiting the high redundancy of the observed surfaces. Monserrat and Crosetto (2008) list typical data analysis procedures required to carry out deformation measurements along with their benefits and limitations. 
Examples of these include: creating a polynomial surface or mesh from different epochs and comparing their shape over time; identifying primitive objects and comparing their movement over time; and determining volumetric change by taking cross-sections, for example Olsen (2009).

Literature directly relevant to the work described in this paper investigates how deformation has been successfully measured in an accurate and precise way. Kopáčik et al (2013) and Erdélyi et al (2014) were able to detect millimetre levels of accuracy and precision for deformation measurements of a bridge and roof respectively using planar surfaces by applying single value decomposition. Laefer et al (2014) were able to detect cracks that were $5 \mathrm{~mm}$ wide with a precision of $1 \mathrm{~mm}$ by using plane fitting techniques of masonry walls, which is similar to the structure scanned in this paper. Puente et al (2012) used mobile TLS to evaluate geometrical deformations of a concrete arch shaped underpass by analysing the arch profiles to achieve deformation measurements between 1-5 $\mathrm{mm}$. The application of TLS for measuring tunnel deformations, which is of a reasonably similar shape of arches, during and after their construction has been widely used with different types of deformation analysis techniques being developed (Yoon et al, 2009). Most recently Nuttens et al (2014) have used state of the art TLS technologies to scan newly built concrete tunnels and monitor them over a period of time to determine their "ovalization". The authors show how millimetric levels of precision could be achieved and that sub-millimetre deformations could be detected and measured by combining TLS and strain gauge measurements.

Despite millimetric accuracy and precision being achieved in the literature, there is limited discussion on how this "monitoring information" is communicated back to engineers, or other interested parties such as stakeholders, to see the outputs from the TLS measurements, for example: Riveiro et al (2011, 2013); Kang (2012); Erdélyi et al (2014); and Nuttens et al (2014).

\subsection{Close Range Photogrammetry}

Luhmann et al (2013) discuss how CRP can be applied for deformation monitoring, particularly in environments with access and time restrictions. Traditionally the areas to be monitored are targeted within a stable network that contains multiple reference points. CRP has the advantage of rapidly capturing a surface from a low cost mobile camera to generate single points or to generate a point cloud for monitoring to be carried out. This method is used in a wide variety of industries, but it is often overlooked in some applications, particularly for deformation monitoring. For example Jiang et al (2008) highlighted how monitoring of bridges is not routinely carried out using CRP despite showing evidence of investigations carried out. CRP is often deployed with survey targets and TS techniques to support geo-referencing.

Whilst the use of targets in CRP is established as an accurate, precise and reliable measurement technique (Luhmann et al 2013), its use to generate point clouds from natural features is highly dependent on the image quality of those features and the geometry of the network of images captured during the survey. All image-based methods rely on the identification of common points or regions in two or more views with automated matching algorithms determining homologous location. Local image texture is essential for successful matching between images (Baltsavias, 1991). A network adjustment incorporating all information, termed a bundle adjustment (Granshaw, 1980), is used to simultaneously estimate 3D locations camera positions and to calibrate the system. In structural monitoring applications, which often happen in dark cluttered environments, key challenges centre on image geometry and image quality. Image geometry requires careful selection of camera views to make sure that every feature appears on at least two convergent views. Image quality is highly 
dependent on being able to illuminate all of the features of interest at the time the photography is taken, for example the arches imaged in this paper were illuminated by electronic flash.

The purpose of this paper is to explore methods of monitoring without the need to attach any type of monitoring target to the structure. The development and widely accessible software for image matching and 3D reconstruction has provided an ideal opportunity to investigate an "ad hoc" method of acquiring a point cloud and comparing it to the output of TLS to investigate its potential as a monitoring tool for this environment. One such example is Structure-from-Motion (SfM) (James and Robson, 2012) which has been applied to a variety of geoscience applications as an alternative to using the more expensive and less portable TLS system. SfM is capable of producing comparable levels of point cloud quality to TLS but requires significant post-processing in comparison to TLS, e.g. Riveiro et al (2013) and Westoby et al (2012). Unlike TLS, which is a polar measurement technique, CRP will fail when a feature of interest is only visible in one image due to occlusion in other images in the network. These aspects are highlighted in Section 3.

\section{The London Bridge Station Arches Case Study}

London Bridge Station is currently undergoing major redevelopment as part of the Thameslink Programme. The station was constructed on a series of masonry arches, built between 1836 and the end of the 19th century. The station's most recent remodelling comprised of 6 through tracks and 9 terminating tracks. The new development will comprise of 9 through and 6 terminating tracks as well as the train shed roof being removed. Due to the planned construction work, many typical railway infrastructures are required to be monitored continuously throughout the project, in particular the railway track and arches. This paper focuses on the monitoring of the masonry arches using traditional TS survey, which also provided geo-referencing for TLS and CRP methods.

\subsection{TS monitoring setup}

The masonry arches are located at street level directly below the platform level of the station. The arches are required to be monitored prior to and during various stages of demolition and construction works. The initial arch monitoring specification was designed to monitor movements during the deconstruction of the old train shed roof overhead. Manual monitoring, which includes prism measurement in arch arrays, has been implemented here on a daily and weekly basis depending on proximity to works and their nature. Automated monitoring has not been applied at arch level to date due to restricted sight lines and continuing changes to work progress.

Monitoring prisms were installed at $5 \mathrm{~m}$ arrays through the arch at the crown and springing points (see Figure 2), whilst reference prisms were located outside the predicted impact zone. Reference prisms were therefore taken to define a stable co-ordinate system for each monitoring epoch.

A Leica TS15 total station was used for the manual monitoring and geo-referencing. The capabilities of the instrument, as quoted by the manufacturer, are 1 " for horizontal and vertical readings and $1 \mathrm{~mm}$ $+1.5 \mathrm{ppm}$ for distance measurements to a prism (Leica Geosystems, 2010). According to the monitoring specification for this project, all targets located on the arches were required to be monitored with an accuracy of at least $+/-1 \mathrm{~mm}$. 


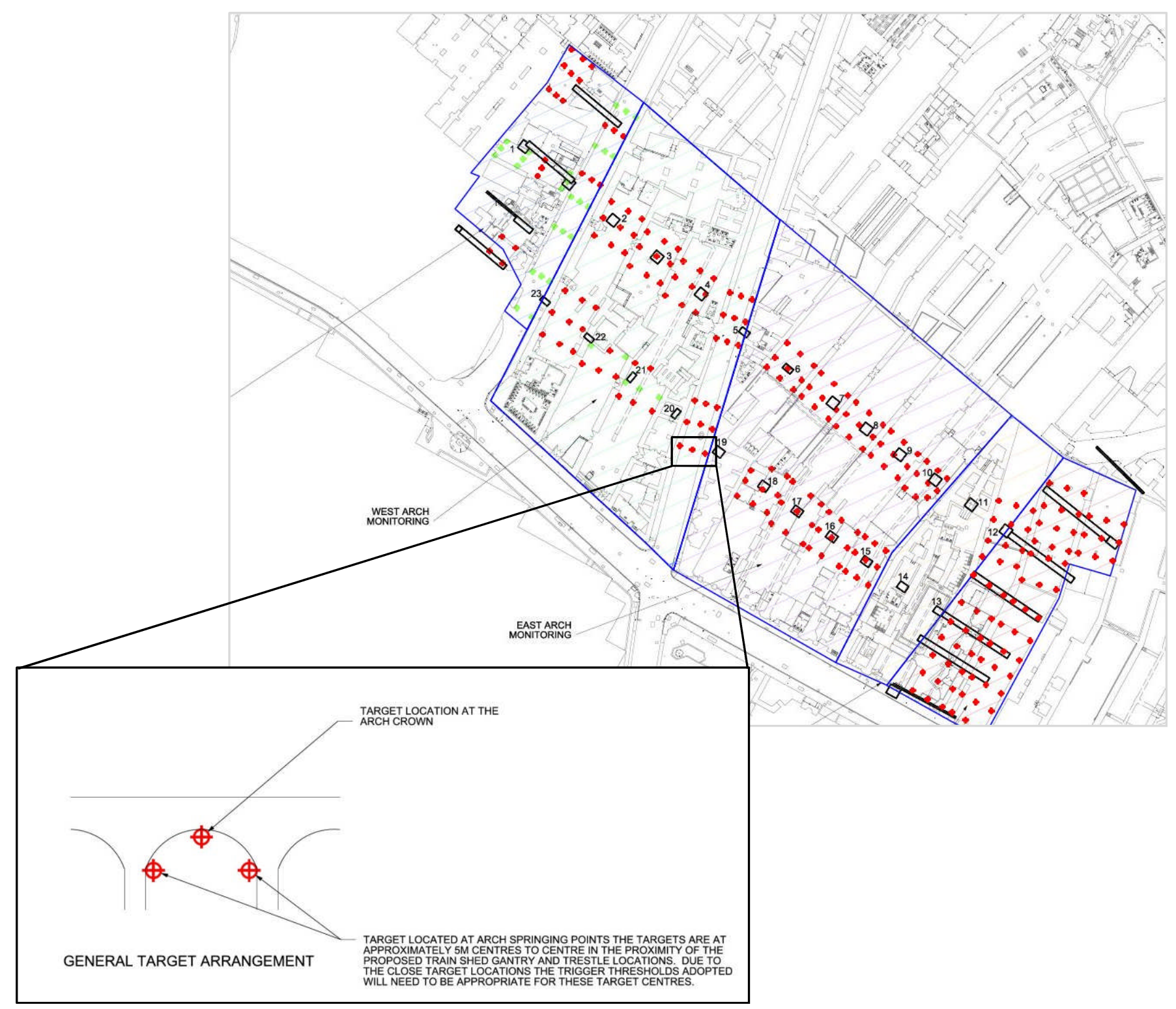

Figure 2 - General target arrangements of arch monitoring

The quality of the network design for this site is affected by the limited lines of sight to the reference prisms through the long and narrow arches, which is similar to that of a tunnelling case. Ideally reference targeting would be placed evenly across the network outside the impact zone, but this would be too invasive and uneconomical in this case. The trade-off is a weaker survey network geometry and lower accuracy when calculating the relative movement of the monitoring prisms. Analysis was carried out to assess the geometric strength of the network using MicroSurvey STAR*NET V7 ${ }^{1}$, a least squares based network processing and analysis tool. A pre-analysis of a test area of the arches, shown in Figure 4, was carried out. Figure 3 shows the reference (triangles) and monitoring points (squares) in the local monitoring network, including predicted confidence level error ellipses for key target prism locations. For the purposes of this case study error ellipses have been generated based on a 95\% confidence level.

\footnotetext{
${ }^{1}$ http://www.microsurvey.com/products/starnet/
} 


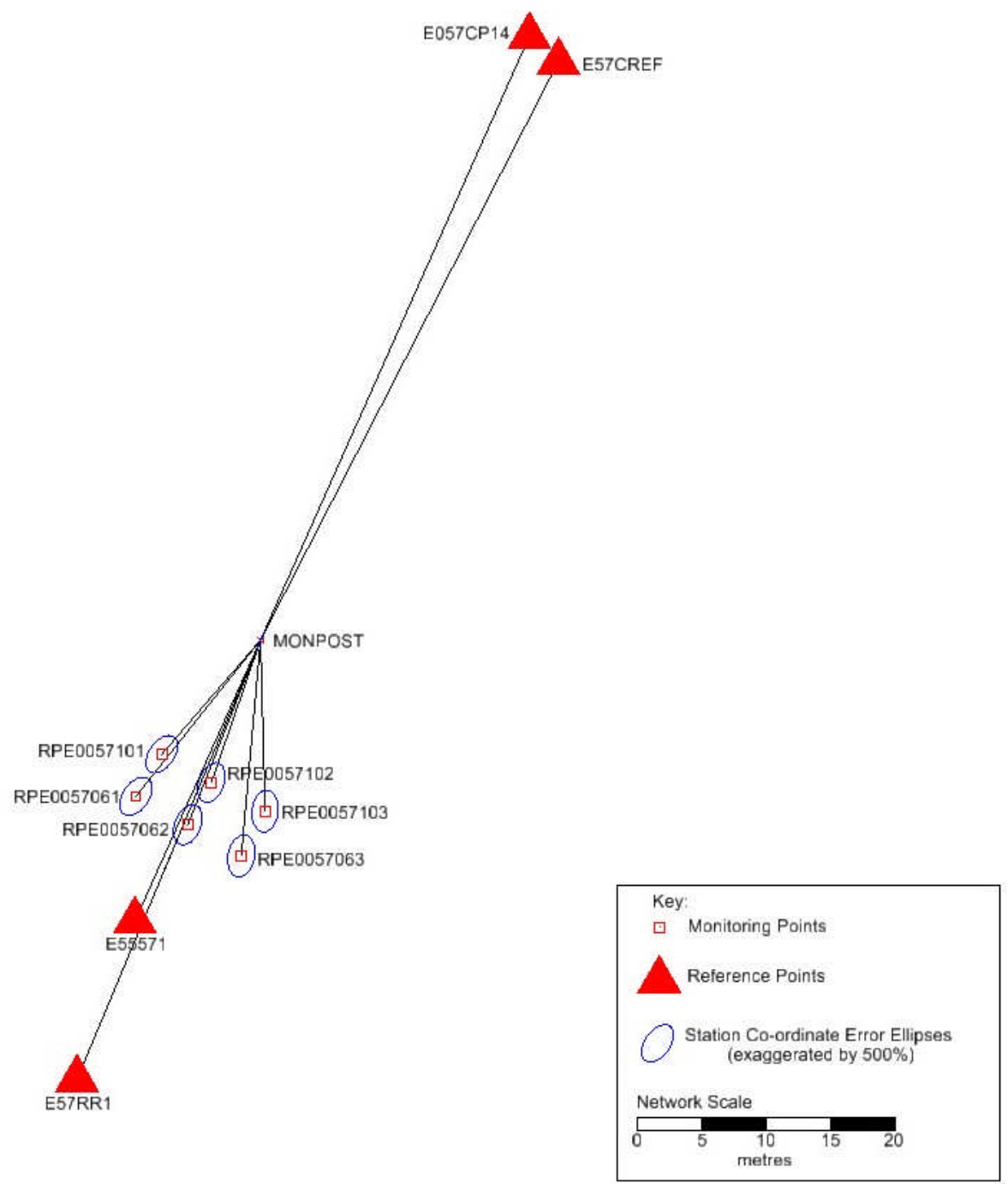

Figure 3 - Network plot of TS15 survey of test area with error ellipses

The standard deviations in Table 1 represent the precision of the monitoring target prism co-ordinates at $2 \sigma$.

\begin{tabular}{|c|c|c|c|}
\hline \multirow{2}{*}{$\begin{array}{c}\text { Monitoring } \\
\text { Point }\end{array}$} & \multicolumn{3}{|c|}{$\begin{array}{c}\text { Point co-ordinate standard deviation } \\
(\mathbf{m m})\end{array}$} \\
\cline { 2 - 4 } & E & N & El \\
\hline RPE0057061 & 1.0 & 1.2 & 1.0 \\
\hline RPE0057062 & 0.9 & 1.3 & 1.0 \\
\hline RPE0057063 & 0.9 & 1.3 & 1.0 \\
\hline RPE0057101 & 1.0 & 1.2 & 1.0 \\
\hline RPE0057102 & 0.9 & 1.3 & 1.0 \\
\hline RPE0057103 & 0.8 & 1.3 & 1.0 \\
\hline
\end{tabular}

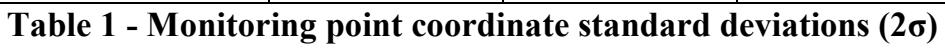

The standard deviations of the monitoring points remain at the $1 \mathrm{~mm}$ level. The network plot shows the error ellipses for the monitoring points have semi-major axis and semi-minor axis which are up to 
$3 \mathrm{~mm}$ and $2 \mathrm{~mm}$ respectively. A survey of this type was carried out at each monitoring epoch to provide updated co-ordinates for the monitoring points.

Subsequent to the train shed deconstruction, 1D levelling was included in the manual monitoring to supplement the 3D observations during the piling works within the arches. The levelling network was linked to an external GPS reference network outside of the impact zone. This linkage includes a set of total station observations to define the targets within the site co-ordinate system. The levelling was partly instigated due to the logistical restrictions (access time and sight-lines during piling works) and partly as an alternative system to validate the movement in order to meet a requirement in the works specification. The co-ordinates of the monitoring points were used to geo-reference the targets used for TLS to enable inter-comparison with the levelling data.

Despite a predicted heave of up to 30mm during the removal of the train shed roof, no differential movement was detected. However during subsequent piling works, the manual prism based monitoring showed that some settlement in the arches was occurring. At this point the engineers required an additional survey method to validate this movement as well as a more detailed inspection to determine how the arch structures were moving whilst the works continued. Additional precise levelling bolts were added to the monitoring regime, and as the area was also being tested for TLS, localised scanning of the arches undergoing deformation was added to the techniques used for validation.

For the analysis discussed in this paper an area defined by 2 prism arrays in the arches was chosen to compare the results from the manual monitoring to the TLS monitoring, with their co-ordinates from manual monitoring used to define a common co-ordinate system against the reference points shown in Figure 3 and Figure 4. 


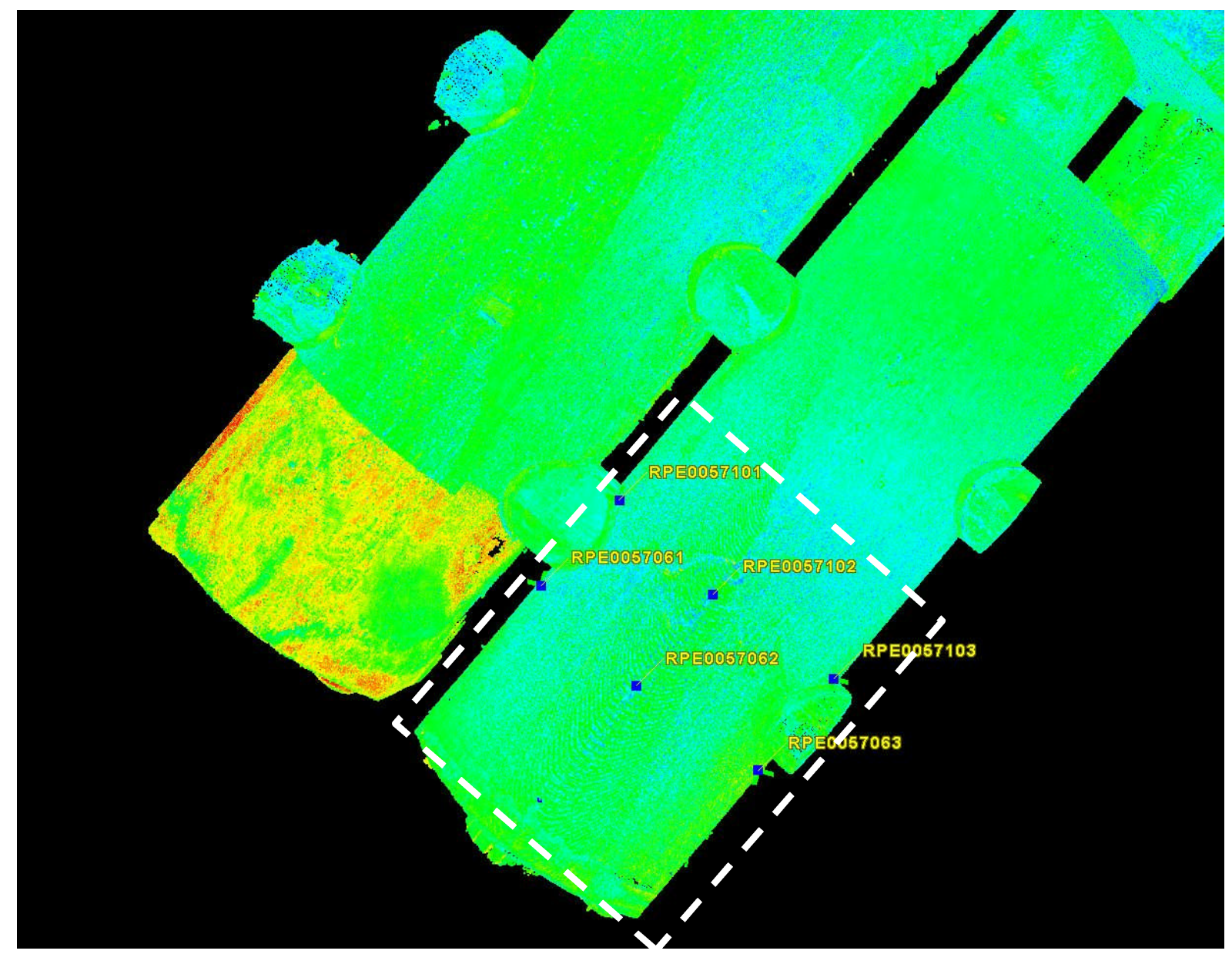

Figure 4 - Test area (white) with monitoring prism arrays (blue): approximately $5 \mathrm{~m} \times 12 \mathrm{~m}$ long

\subsection{TLS monitoring setup}

A FARO Focus ${ }^{3 \mathrm{D}}$ scanner was available on site. Originally purchased by the Design and Build Contractor to illustrate topographic survey updates, this instrument was selected to monitor the set of arches subject to movement. The Focus ${ }^{3 \mathrm{D}}$ is a phase based scanner which has vertical and horizontal ranges of $305^{\circ}$ and $360^{\circ}$ respectively. According to the manufacturer's specification, the maximum operating range is $120 \mathrm{~m}$ with a ranging error of $\pm 2 \mathrm{~mm}$ (FARO, 2013). Chow et al (2012) carried out a point-based self-calibration of this system and was able to show the ranging precision was better than $2 \mathrm{~mm}$ before and after the calibration procedure, agreeing with the manufacturer's specification.

Scanning was carried out on four separate occasions (epochs) over an eight week period following the detection of initial movement until the settlement appeared to have stopped. The number of scans for each epoch varied due to the limited access to the site whilst works continued. Scanned surfaces were typically at ranges of 5 metres from the scanner with a point spacing of $4 \mathrm{~mm}$. To increase the accuracy of the registration of scans for a particular epoch, sphere targets were used. Scan-to-scan registration was carried out in Faro Scene version 5.1 ${ }^{2}$. Results from the TS monitoring were utilised in order to select stable areas so that each epoch could be registered to the "Epoch 0" baseline dataset with the Iterative Closest Point (ICP) algorithm (Besl, 1992) available within Leica Cyclone ${ }^{3}$ version 8.1 software. Stable areas comprised of the base of the arches up to a height of up to approximately 1

\footnotetext{
${ }^{2}$ http://www.faro.com/products/faro-software/scene/overview

${ }^{3}$ http://hds.leica-geosystems.com/en/Leica-Cyclone_6515.htm
} 
metre distributed across the entire point cloud. Registrations indicated by the software shows an average RMS registration of $7 \mathrm{~mm}$.

\subsection{CRP setup}

To compare the outputs of TLS without the use of targets and to show the potential of other applications for deformation monitoring in a railway environment, CRP was applied to the arch at one of the epochs. A Nikon D3200 camera was used with a calibrated wide angle $16 \mathrm{~mm}$ fish-eye lens to capture a network of images of a test section of the arch. The images were then corrected for distortion using UCL's Lens Distortion Correction (LDC) software and a fish-eye correction model. The corrected images were run through Visual SFM ${ }^{4}$ version 0.5.22 - an open source Structure from Motion bundle adjustment based tool - to carry out feature detection and full pairwise image matching. The dense 3D reconstruction function was then run to produce a point cloud of the area. The CRP point cloud was then scaled and compared to the TLS point cloud. 3D distances between common features within the CRP and TLS point cloud were used to compute a scale factor for the CRP point cloud. Once scaled, the same reference points could be used to approximately align the CRP data. Fine registration was then achieved using CloudCompare ${ }^{5}$ version 2.4 software based on the same stable regions as originally used to inter-compare the TLS data. This process results in a global error of $3 \mathrm{~mm}$. Section 4.5 details some of the tests carried out to validate the CRP results.

\section{Results and Analysis}

4.1. Comments on Workflow

The workflow for each of the methods, including design of the sensing network through to communicating measurement data to engineers, is shown in Table 2. The TS workflow established the geo-reference for the system and was able to co-ordinate some half dozen targets which were essential to both CRP and TLS geo-referencing. Without the use of TS both CRP and TLS would only have been capable of determining relative movement between epochs as opposed to absolute movement. Similarly if TS was used on its own, only a few dozen points could have been recorded rather than the complete surface geometry obtainable from either TLS or CRP.

\footnotetext{
${ }^{4} \mathrm{http}: / /$ www.ccwu.me/vsfm/

${ }^{5} \mathrm{http}: / / \mathrm{www} \cdot$ danielgm.net/cc/
} 


\begin{tabular}{|c|c|c|c|c|c|c|c|c|c|}
\hline Instrumentation & \multicolumn{9}{|c|}{ Methodology } \\
\hline & Pre-analysis & $\begin{array}{c}\text { Data } \\
\text { Capture }\end{array}$ & \multicolumn{4}{|c|}{ Data Processing } & \multicolumn{2}{|c|}{ Outputs } & $\begin{array}{c}\text { Communication } \\
\text { and Reporting }\end{array}$ \\
\hline $\begin{array}{l}\text { TS (medium } \\
\quad \text { cost) } \\
\text { Several setup } \\
\text { positions }\end{array}$ & \multirow{3}{*}{$\begin{array}{l}\text { Simulation and } \\
\text { design of } \\
\text { sensing } \\
\text { network } \\
\text { against } \\
\text { requirements }\end{array}$} & \multirow{3}{*}{$\begin{array}{c}\text { Instrument } \\
\text { and target } \\
\text { placement } \\
\text { Repetitive } \\
\text { data capture }\end{array}$} & Orientation & $\begin{array}{c}\text { Network } \\
\text { adjustment }\end{array}$ & & & \multicolumn{2}{|c|}{ Discrete points } & $\begin{array}{l}\text { Web-based } \\
\text { reporting of } \\
\text { movement in } \\
\text { tabular and } \\
\text { graphical } \\
\text { format }\end{array}$ \\
\hline $\begin{array}{l}\text { TLS (high cost) } \\
\text { Several scan } \\
\text { positions }\end{array}$ & & & Registration & $\begin{array}{c}\text { Network } \\
\text { adjustment } \\
\text { (optionally } \\
\text { with geo- } \\
\text { referencing) }\end{array}$ & & & $\begin{array}{l}\text { Point } \\
\text { cloud }\end{array}$ & $\begin{array}{l}\text { Output } \\
\text { surface } \\
\text { mesh }\end{array}$ & $\begin{array}{l}\text { Deformation } \\
\text { displacement } \\
\text { maps/extracted } \\
\text { profiles }\end{array}$ \\
\hline $\begin{array}{l}\text { CRP (low cost) } \\
\text { Many images }\end{array}$ & & & $\begin{array}{l}\text { Image } \\
\text { correction } \\
\text { and image } \\
\text { matching }\end{array}$ & $\begin{array}{l}\text { Network } \\
\text { adjustment } \\
\text { (optionally } \\
\text { with scale } \\
\text { and geo- } \\
\text { referencing) }\end{array}$ & $\begin{array}{l}\text { Dense } \\
\text { recon- } \\
\text { structi } \\
\text { on }\end{array}$ & $\begin{array}{l}\text { Scale/ } \\
\text { Geo- } \\
\text { refere } \\
\text { nce }\end{array}$ & $\begin{array}{l}\text { Point } \\
\text { cloud }\end{array}$ & $\begin{array}{l}\text { Output } \\
\text { surface } \\
\text { mesh }\end{array}$ & $\begin{array}{l}\text { Deformation } \\
\text { displacement } \\
\text { maps/extracted } \\
\text { profiles }\end{array}$ \\
\hline
\end{tabular}

Table 2 - Methodology workflow of TS, TLS \& CRP for monitoring purposes

Despite having similar data acquisition times on site, CRP required significantly more backoffice time to get to the point cloud output due to the number of steps required in the workflow. It was also found that in order to process images encompassing one arch required an extensive computer processing power (a high end PC with 8 processor cores and 64GB RAM). The CRP output is dependent on the local surface texture and the overlapping network of convergent images that can be captured across the surface. The major limitation, with current CRP field technology is that it is a passive sensor and therefore results cannot be realised and checked until the final step. In contrast TLS is an active sensor providing close to real-time output of point cloud and if areas have been missed, it can be seen immediately and scanned.

The skill set required for these survey methods differs. CRP techniques require a specialised skill set to capture the image network necessary to produce the optimum output through the workflow. Once an accurate point cloud has been acquired and registered the data processing workflow is relatively similar and commonly used by those working in the engineering surveying industry. The attraction of CRP is the realtively low cost and widely available digital camera as a sensor, but this is offset by the skills needed to capture the data. As mobile computing improves, automation of CRP capture may well reduce the required operator skills. As TLS can measure from a single station, the data capture process is somewhat easier being one of obtaining full coverage rather than the complete multi-view coverage required by CRP. Despite this, if the scanner was not available, it is a relatively high cost sensor and less readily available if bespoke validation tools are required. In contrast, CRP uses low cost cameras which are more readily availble. 


\subsection{Manual Monitoring Results}

Figure 5 shows a graph of one of the monitoring prism readings, relative to the baseline readings in the $\mathrm{Z}$ direction, within the test area of the arches. This monitoring information is provided to the engineer via a web-based reporting system that highlights change through the use of pre-set trigger threshold alarms. Typically a traffic light warning system of green, amber, and red alarms are set by the engineers to describe the levels of acceptable movement, which is based on the predicted levels of movement. In this study the trigger thresholds are defined in terms of settlement where green $=$ $\leq 10 \mathrm{~mm}$ settlement, amber $=>10 \mathrm{~mm}$ and $\leq 15 \mathrm{~mm}$, red $=>15 \mathrm{~mm}$. The time series of the graph shows the monitoring data acquired before, during and after the piling works began. The green arrows indicate epochs at which TLS was carried out. In this case the decision made by the engineering team was to increase the monitoring frequency and to verify the survey network link to external georeferenced points whilst continuing the plan of work.

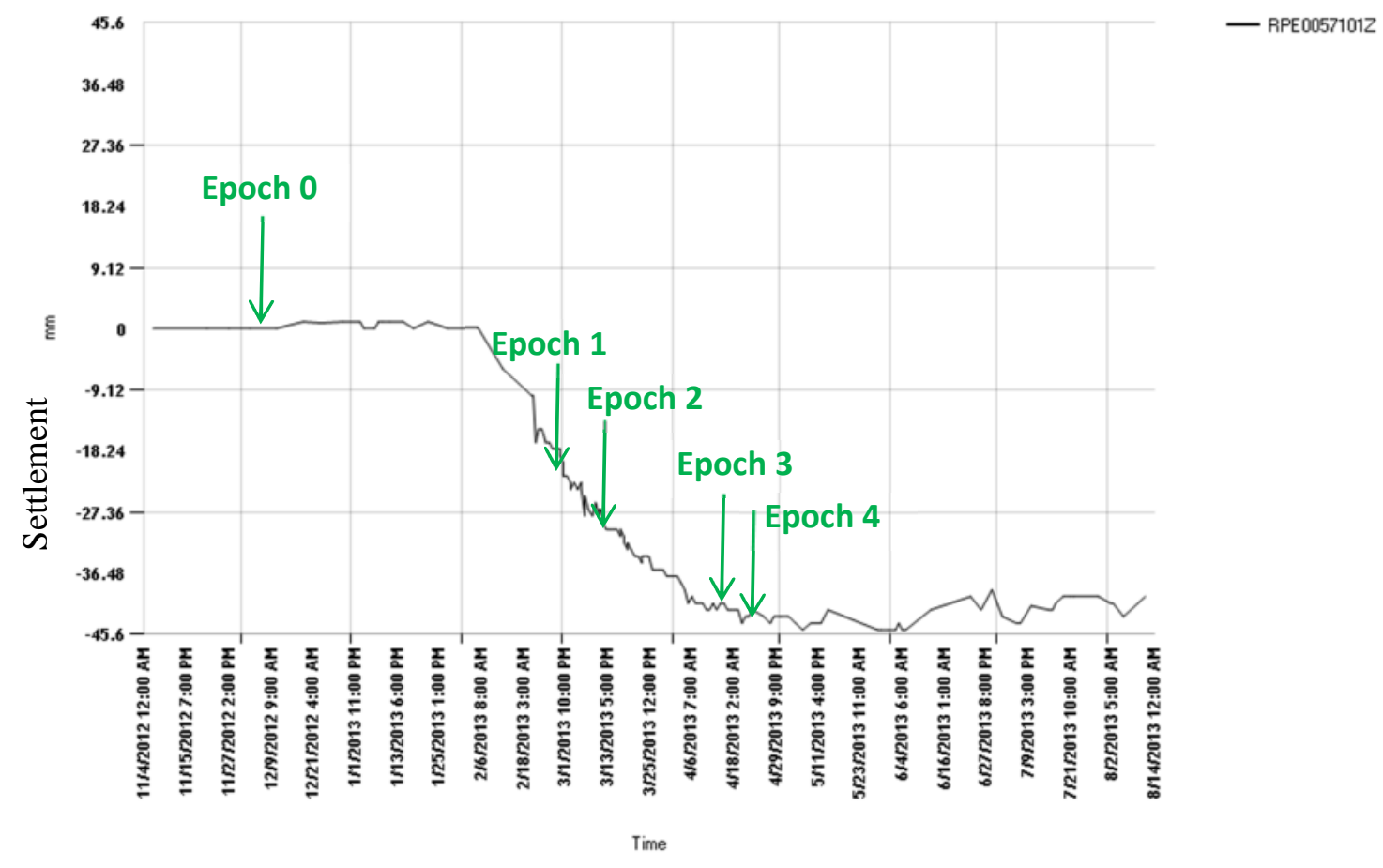

Figure 5 - Graphical monitoring results from target RPE0057101 (mm)

Table 3 shows monitoring data from the two arrays of prisms in the test area (refer to Figure 4). The data shown in red are the recorded changes shown in Figure 5. 


\begin{tabular}{|c|c|c|c|c|c|c|}
\hline \multicolumn{7}{|c|}{ Array 1 (left to right) (mm) Movement in $\mathrm{Z}$ axis (-ve value is a settlement) } \\
\hline $\begin{array}{c}\text { Epoch/Target } \\
\text { ID }\end{array}$ & RPE0057061 & $\begin{array}{l}\Delta \text { movement } \\
\text { RPE0057061 }\end{array}$ & RPE0057062 & $\begin{array}{l}\Delta \text { movement } \\
\text { RPE0057062 }\end{array}$ & RPE0057063 & $\begin{array}{l}\Delta \text { movement } \\
\text { RPE0057063 }\end{array}$ \\
\hline 0 & 1 & & 1 & & 1 & \\
\hline 1 & -22 & -23 & -14 & -15 & -2 & -3 \\
\hline 2 & -31 & -9 & -19 & -5 & -2 & $\mathbf{0}$ \\
\hline 3 & -41 & -10 & -23 & -4 & -1 & 1 \\
\hline 4 & -42 & -1 & -22 & 1 & -2 & -1 \\
\hline 5 & -42 & $\mathbf{0}$ & -22 & $\mathbf{0}$ & -1 & 1 \\
\hline 6 & -43 & -1 & -22 & $\mathbf{0}$ & -2 & -1 \\
\hline
\end{tabular}

\begin{tabular}{|c|c|c|c|c|c|c|}
\hline \multicolumn{7}{|c|}{ Array 2 (left to right) (mm) Movement in $\mathrm{Z}$ axis (-ve value is a settlement) } \\
\hline $\begin{array}{c}\text { Epoch/Target } \\
\text { ID }\end{array}$ & RPE0057101 & $\begin{array}{l}\Delta \text { movement } \\
\text { RPE0057101 }\end{array}$ & RPE0057102 & $\begin{array}{l}\Delta \text { movement } \\
\text { RPE0057102 }\end{array}$ & RPE0057103 & $\begin{array}{l}\Delta \text { movement } \\
\text { RPE0057103 }\end{array}$ \\
\hline 0 & 1 & & 0 & & 1 & \\
\hline 1 & -24 & -25 & -14 & -14 & -2 & -3 \\
\hline 2 & -33 & -9 & -19 & -5 & -2 & $\mathbf{0}$ \\
\hline 3 & -42 & -9 & -23 & -4 & -2 & 0 \\
\hline 4 & -43 & -1 & -22 & 1 & -2 & $\mathbf{0}$ \\
\hline 5 & -43 & 0 & -22 & 0 & -2 & 0 \\
\hline 6 & -44 & -1 & -22 & $\mathbf{0}$ & -2 & $\mathbf{0}$ \\
\hline
\end{tabular}

Table 3 - Numerical manual monitoring results of arches including change in movement between epochs (mm)

Between the 2 corresponding arrays of prisms, the results show a consistent set of movement. Significant change in movement is detected between epochs 1 and 3 (with changes of up to $25 \mathrm{~mm}$ with respect to the first epoch), which then levels off by epoch 4 . The left side of the arch is settling up to $-43 \mathrm{~mm}$ ( $\mathrm{Z}$ direction), whilst the right side of the arch remains relatively stable. The crown of the arch shows a gradual settlement reaching $23 \mathrm{~mm}$ which then stops by epoch 4 . The results show discrepancies indicating settlement of up to $20 \mathrm{~mm}$ between the crown and left of the arch. This settlement is considered highly significant, being an order of magnitude larger than the $3 \mathrm{~mm}$ maximum deflection observed to the right of the arch and when compared to the $1.3 \mathrm{~mm}$ maximum coordinate standard deviations $(2 \sigma)$ estimated from the survey network (see Table 1 ).

\subsection{TLS Results}

Direct measurement to prisms with TLS is unreliable with the FARO system used in this study. However the dense and relatively complete surface information that TLS provides allows an areawide displacement map to be computed. Data from the dense deformation displacement map can be used for inter-comparison. Profiles were also produced to provide the engineers with a more conventional understanding of the observed movement. 
The deformation displacement maps in Figure 6 were produced by comparing the meshed surfaces for each epoch to the baseline measurement in Geomagic Studio \& Qualify $2013^{6}$. The 3D deviation tool available within the software is able to calculate the deviations as the shortest distance from the test object to the reference object. The deformation displacement maps shown are in plan-view for each of the epochs compared to Epoch 0 . The scale of displacement analysed is $+0.05 \mathrm{~m}$ to $-0.05 \mathrm{~m}$. The colours are representative of the movements classed in the original trigger threshold values set for the monitoring of the arches during the deconstruction of the train shed roof (see Section 4.2). The grey areas highlight where there was insufficient data overlapping between the scans. Each of the maps shows a black spot towards the north of the test area, implying a settlement of greater than $50 \mathrm{~mm}$. After further investigations, this was found to be an anomaly where there was a gap in the baseline data due to occlusions caused by lighting fixtures on the crown of the arch.

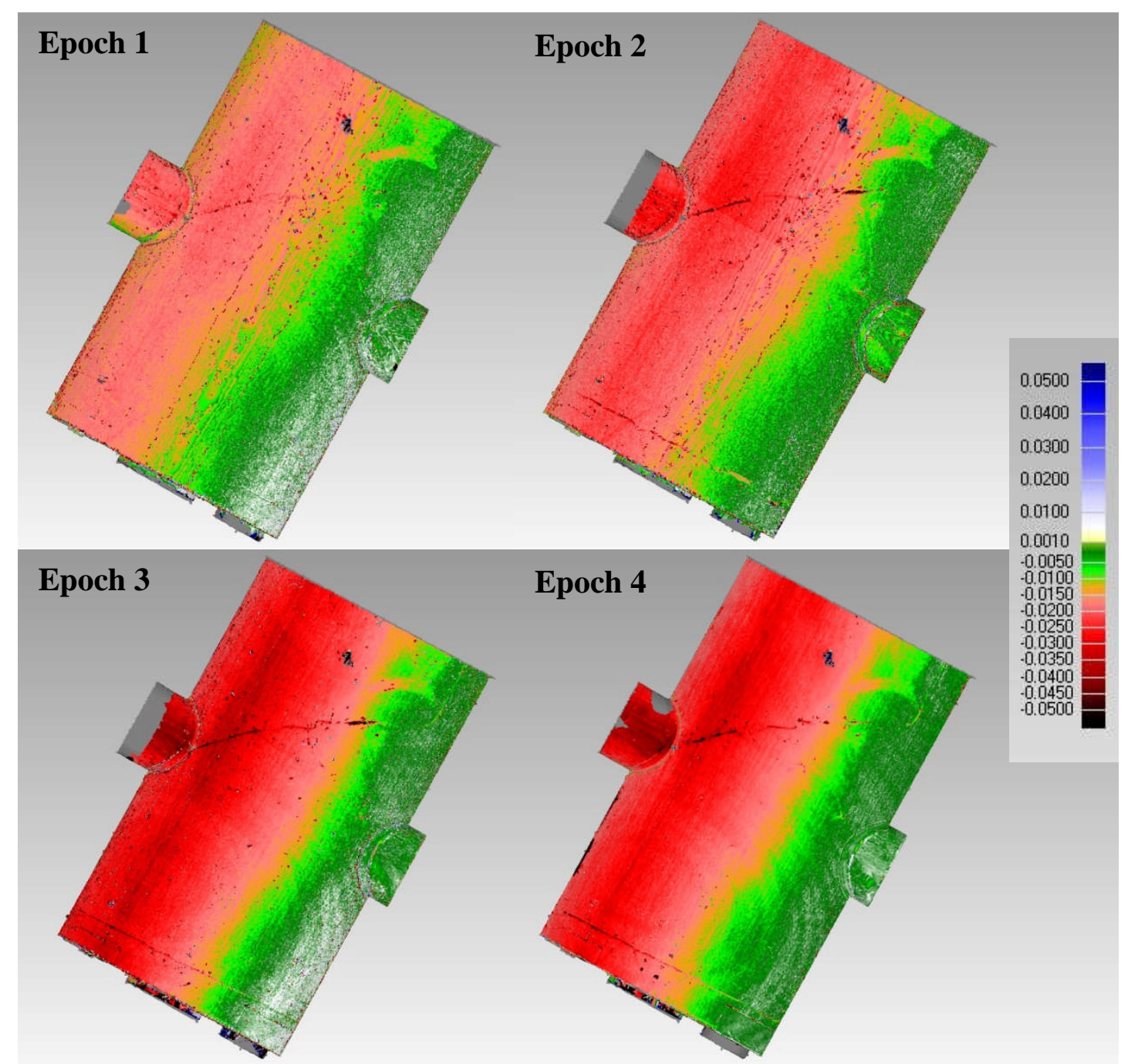

Figure 6 - Deformation Displacement maps of a 5x12 metres area of local detail between Epochs 1 and 4 (plan view, deflections given in metres)

\subsection{TS and TLS Analysis}

The colour scaled surface deformation displacement map highlights the settlement of the arches. Results agree with the observations from the manual monitoring data. In this case the scan data is

${ }^{6}$ http://geomagic.com/en/products/studio/overview/ 
independent of the TS monitoring survey and provides more detail of the surface of the arches. The accuracy of the displacement map is dependent on the ICP registration between the epochs calculated in Cyclone version 8.1. In Figure 6, where data between epochs has been compared in Geomagic Studio 2013, it is readily apparent that significant areas of the right hand portion of the arch show typical discrepancies of the order of $1 \mathrm{~mm}$ demonstrating the validity of the registration. The surface displacement map is significant for the engineers as it firstly validates the manual monitoring results, but also provides more information about the structure for the engineer to carry out further analysis. The colours have been selected to highlight the various trigger alerts that would be set off if this was developed into a reporting system. However, in this instance, the only way of providing numerical information of the deformation is through the coloured scale.

In order to directly compare results from TLS to the prism monitoring, an attempt to extract the discrete information from the surface model in the vicinity of each prism was made. The discrepancies between the movement from the manual readings and expected readings from the scan data show variations of between $1 \mathrm{~mm}$ and $20 \mathrm{~mm}$ highlighting the weakness of this method, despite $5 \mathrm{~mm}$ point spacing of the surface in proximity to the prism. An alternative method would be to apply local fitting to the TLS data to compare them to the TS dataset. However prism locations have been selected by the engineer according to the arch geometry and do not easily support local fitting (see Figure 7) such as the plane fits used in Section 4.5, necessary to ensure accurate results. In order to make direct comparisons to the prisms, physical adapters to prisms or spheres would need to be attached in the place of each prism. Alternatively a common target incorporating a prism and checkerboard could be assigned.
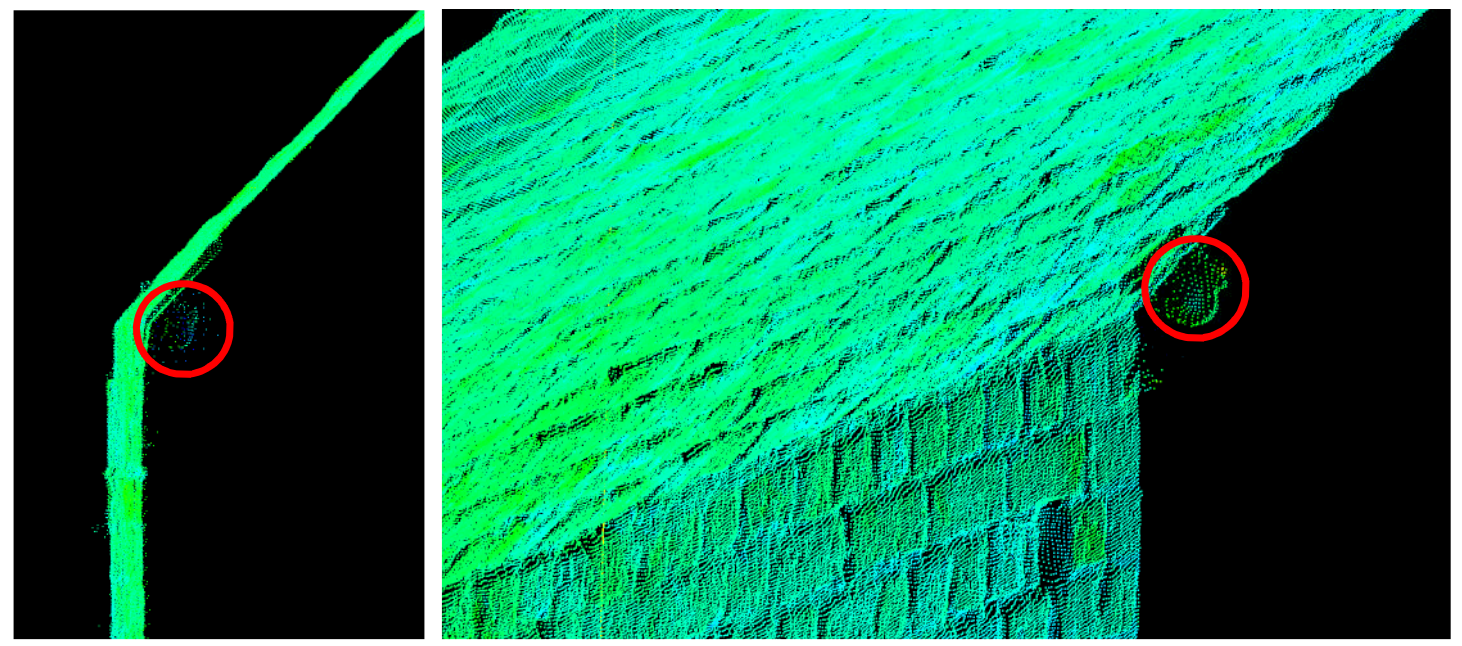

Figure 7 - Side (left) and perspective (right) view from point cloud of prism (circled in red) aligned to springing point of arch

Profiles through the arch at each epoch were manually extracted using CAD software. The profiles were constructed at approximately the same position as the prism arrays. An example is shown in Figure 8. Even though the exact location of the prisms cannot be identified in the scans, the difference in distance measurements provides a way of visualising discrete measurements across the array. In this example, the profile shows a set of six discrete deformation measurements that have been extracted from the scans compared to the three prism based measurements available. Given the observed change in this test area, the engineer found that being able to choose both profile locations and the number of data points to be extracted per profile provided an ideal solution. Some parts of the profile have required more "extraction" than others, particularly when analysing the effect of 
unpredicted movement. This allows the engineers to have flexibility with distinguishing where more localised monitoring was required to take place. This is not a practical solution for prism based monitoring as, in the simplest case, it could result in installing a very large number of prisms which would result in further costs for installation, maintenance and the time required on site. Meanwhile this need for an "extra" set of measurements would not be required if TLS was applied, as the data would contain all this information and could be used on an "as and when needed" basis. This then becomes a balance between being able to extract information to the level of accuracy required and what exact information the engineer requires to see in the reporting.

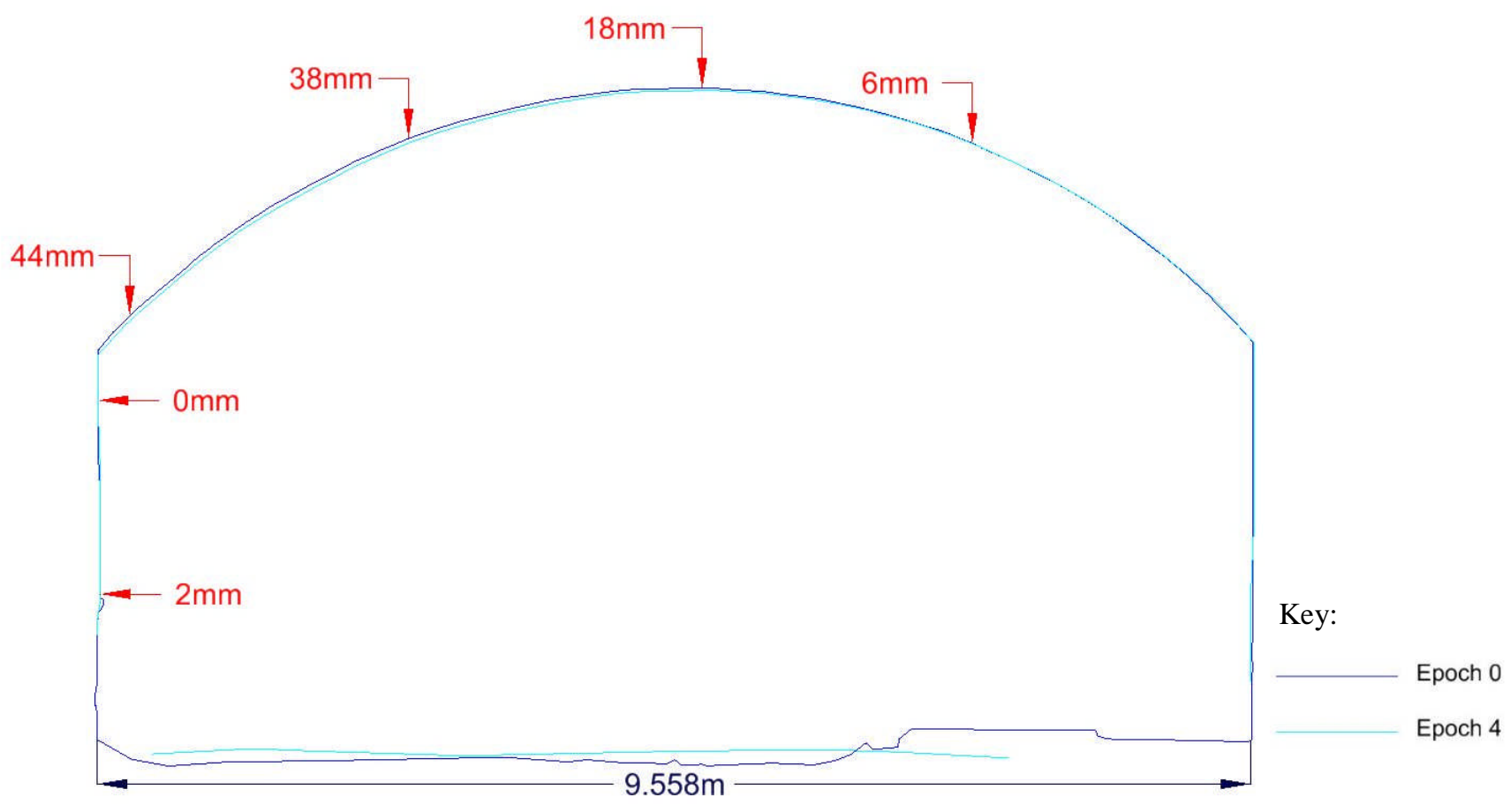

Figure 8 - Profile of arch from TLS data with movement vector dimensions (deflections shown in red)

Geo-referencing in this particular application is challenging due to narrow sight lines. However if it could be improved, the effectiveness and independence from local deformation of the TLS would benefit, since ICP and assumptions of stable surfaces would no longer be needed.

To overcome the requirement of needing a TS and TLS system whilst the need to maintain a high accuracy of survey, manufacturers are producing laser scanning total stations which have the capabilities of reading prism targets accurately and scanning localised surfaces similar to a TLS system, .e.g. the newly released Leica Nova MultiStation MS507. Whilst the scanning time is significantly longer with the first generation of these devices, these types of instruments are likely to reduce the time required when using the TS and TLS system as well as produce discrete point measurements to a target in the arches as well as scan a surface that might require further investigation. A future possibility is in the combination of sensing systems with scanning technology as discussed in Section 2.1, however current methods are not capable of achieving the indoor millimetric measurement accuracies required for this application

\footnotetext{
${ }^{7}$ http://www.leica-geosystems.co.uk/en/Leica-Nova-MS50_103592.htm
} 
In practical terms local referencing on ICP is likely to remain a requirement since strengthening the TS network geometry for accurate geo-referencing in these arches would be challenging due to the long and narrow shape and therefore limited lines of sight for a network solution (see Figure 3). To overcome this, more TS setups would be required which, in turn, would introduce more sources of error which would be propagated through the survey network. Simulation of possible networks could be used to optimise the solution, but initial estimates suggest that magnitude of these errors would be of the same order as the predicted movement levels.

\subsection{CRP vs TLS}

A test area within the arches was chosen to compare the processes and outputs from CRP to TLS. The workflow, shown in Table 2, was used to produce a scaled and registered point cloud of the same arch. An example from the CRP output can be seen in Figure 9.

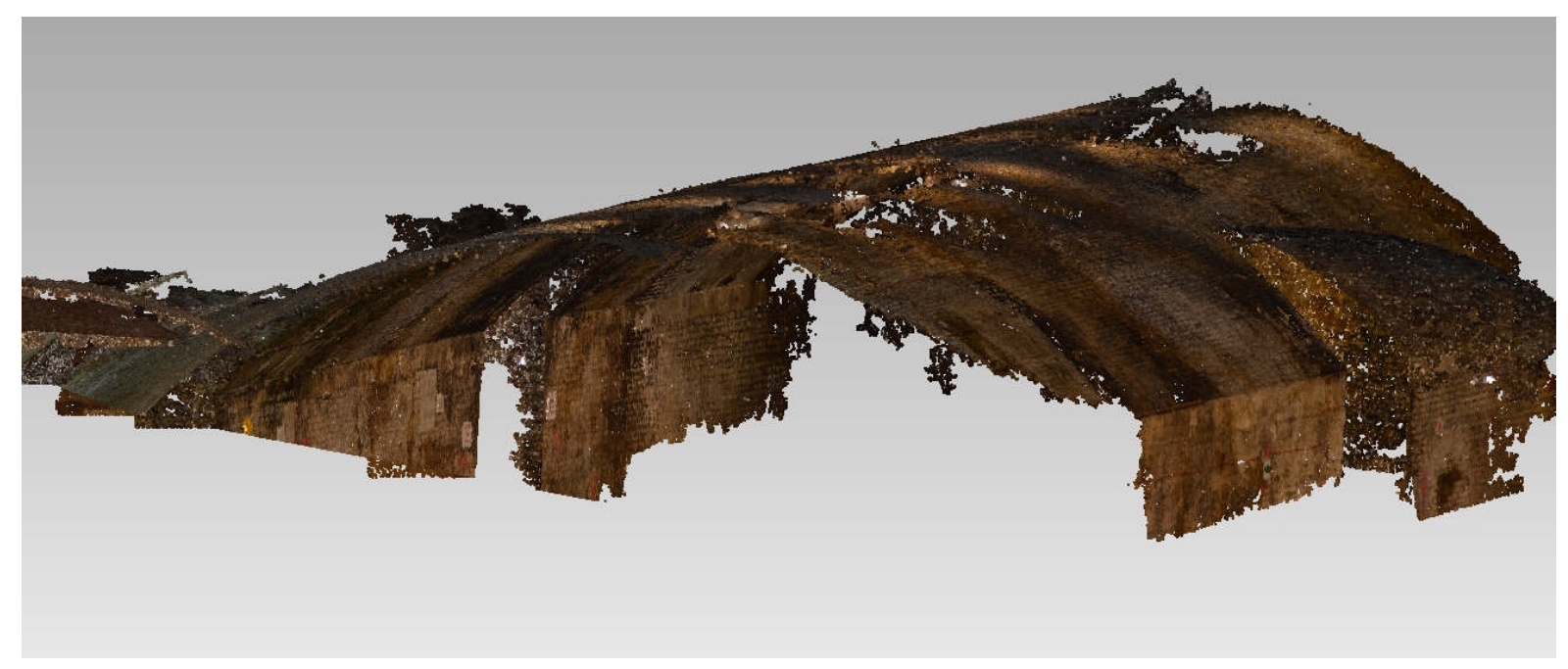

Figure 9 - CRP point cloud of test area of masonry arches

Local comparisons between the TLS and CRP point clouds were carried out by looking at the individual patches and profiles of each of the datasets.

Patches of relatively flat areas in the corresponding point clouds were extracted and compared. By using least squares estimation, planes were fitted to these patches using the software Shapes (Fryer et al, 2002). As the arches are not perfectly planar, the RMS of the residuals following plane fiting provide a relative comparison between the two surveying methods. These are shown in Table 4.

\begin{tabular}{|l|c|c|}
\hline & \multicolumn{2}{|c|}{ RMS of the residuals, normal to the plane (mm) } \\
\hline & TLS & CRP \\
\hline Plane 1 & 7.3 & 11.3 \\
\hline Plane 2 & 2.2 & 3.0 \\
\hline Plane 3 & 1.7 & 1.7 \\
\hline Plane 4 & 3.5 & 3.3 \\
\hline
\end{tabular}

Table 4 - Results from plane fitting using least squares estimation

The RMS of the residuals from the plane fit shows the level of noise of those particular patches from the two sensors and therefore demonstrates the sensitivity of TLS and CRP to detect change. The values for these areas are comparable between the methods and show a millimetric level of noise. The exception is Plane 1, where on closer observation a strip of wood (Figure 10) could be seen attached 
to the extracted region, this small 3D feature with its sharp edges and sharp shadowed edges has been recorded differently by the active TLS and the passive CRP system.

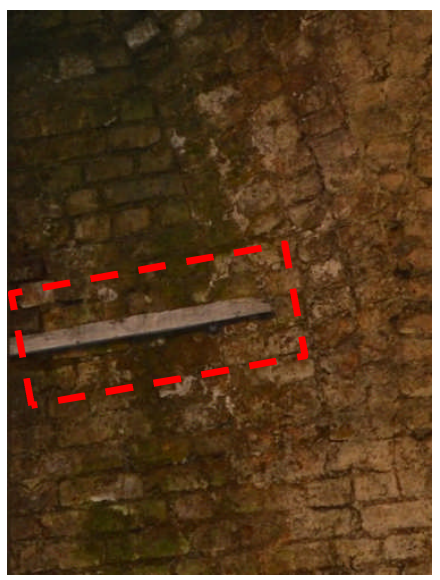

Figure 10 - Image of area used for Plane 1 highlighting wooden object

Figure 11 shows the point cloud used for the "Plane 1" fit using the TLS and CRP data. It shows the visibility of the wooden object in both datasets. In this case the difference in the magnitude of the residuals highlights the spatial sensitivity of the methods to record fine detail, suggesting that the photogrammetric surface is more sensitive to this influence. The profile views from both data sets confirms this (lower image pair in Figure 11).

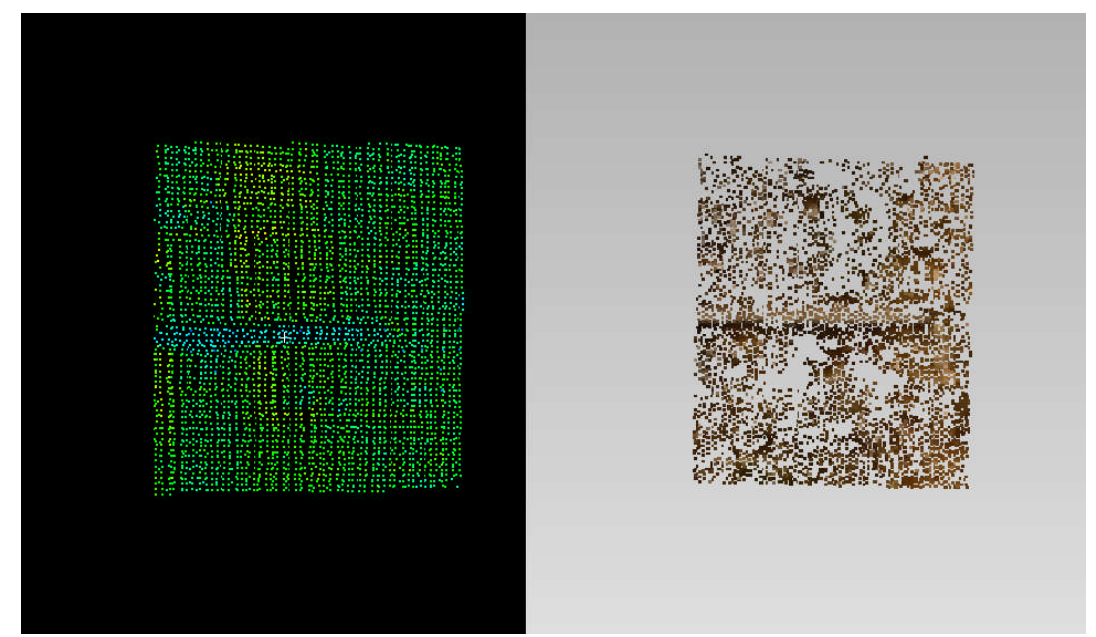




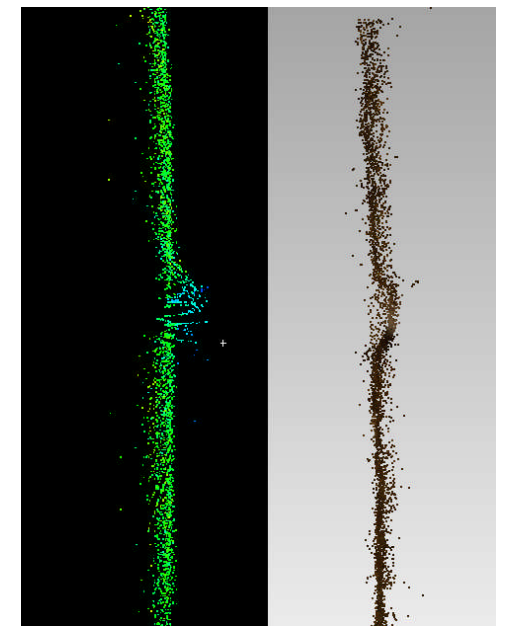

Figure 11 - Front and side profile view of TLS (left) and CRP (right) point cloud for Plane 1

Corresponding profiles of the arch from the CRP and TLS datasets were extracted and compared. An example profile is shown in Figure 12 where the TLS points (which are set as the baseline dataset) are represented in dark blue and CRP in light blue. Discrepancies between two systems along the arch are shown. A close up image of the point clouds of the area highlighted in the grey box is shown in the lower image.

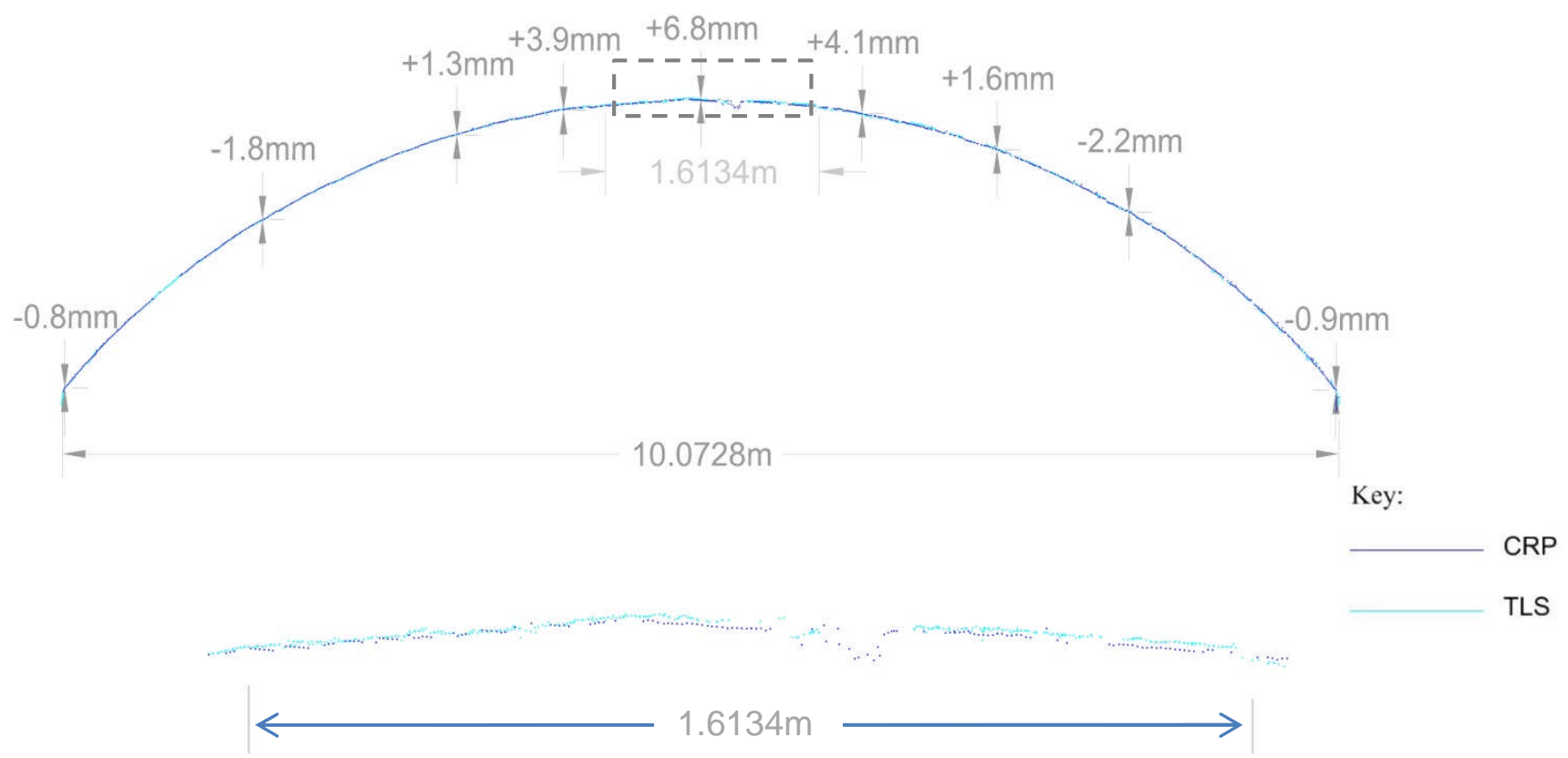

Figure 12 - Comparison of arch profile between TLS and CRP data (in mm)

As the global registration error for the TLS and CRP data was 3mm (Section 3.3), the profile shows a relatively good fit between the two datasets. The springing point of the arch shows a very small discrepancy between the datasets, i.e. less than $1 \mathrm{~mm}$, and correspond to the boundaries of the ICP registration data selection. The level of discrepancy is higher towards the crown of the arch reaching up to $6.8 \mathrm{~mm}$. A possible reason for this discrepancy is the weakness in the photogrammetric image network geometry. Due to the site logistics, it was not possible to take images in proximity to the crown compared to the springing points of the arch. These discrepancy results suggest that this affected the image geometry and subsequently degraded the point cloud output. Therefore further 
work would be required to improve the image network so that sufficient coverage of the crown of the arch can be acquired.

\section{Conclusions and Further Work}

Prism based monitoring, which is conventionally used for monitoring, provides a quick and accurate method of monitoring a set of discrete points across a structure. When settlement occurred in this case study, independent checks of the global quality of the survey data were requested by the engineers. However, due to the economics and logisitics of the site, validating the relative movement became the focus. Unfortunately there was not an opportunity to carry out an independent check with a total station.

TLS was implemented as an independent survey method to validate this movement over a series of epochs. As there was limited time on site to carry out site survey work, TLS proved to be invaluable for capturing a larger volume of surface information of the arches. The movement was validated and communicated to the engineers through surface displacement maps as well as providing $2 \mathrm{D}$ profiles of the arches over a time period. In comparison to traditional survey methods, this was a longer process for validating the movement, but it took less time on site than a global check of the prisms would have taken. TLS also provided the engineers with more complete information about the surface of the structure and the shape of the movements taking place. The main advantage of using this sytem is that the data for a structure can be used in multiple ways, even if they are not all realised in the first instance. For example, this study used the baseline point clouds (required for the as-built surveys) when analysing the movement. This use could not have been predicted when the initial survey was being carried out. Due to the use of TLS in all aspects of survey and the increasing skillsets that surveyors can provide, no extra personnel would necessarily be required to carry out the process and analysis for this particular environment.

A CRP survey was carried out during one of the epochs in order to compare the system to the TLS. The results showed that the point cloud quality was comparable to TLS. However, this process was very time consuming and required intensive processing power. Due to CRP being a passive method of survey, the quality of the data cannot be evaluated (for example missing gaps of the surface) until the end of the workflow. The method is reliant on surface texture unlike TLS. Therefore this method would require a more skilled person to ensure data capture and processing is carried out effectively. As with the historical adoption of TLS, achieving an efficient workflow is a function of technology and training and we would that expect a similar steep increase in technological capability will occur in the relatively low cost CRP domain to make best benefit of its capabilities.

For construction sites and railway sites in particular, logistics and efficiency are just as crucial as the accuracy required when implementing a monitoring system. Table 5 summarises the different methods used in this paper, as well as factors that should be considered by the engineers when implementing monitoring. It can be seen that no single solution is ideal for all monitoring sites and that comprimises must be made depending on the specific site, e.g. expected levels of movement, time available on site for installation/measurement. This case study has highlighted the need for a validation or backup monitoring system in the case of unpredicted movement.

This case study provides an ideal location to test the capabilities of recently released instrumentation which combines total station and laser scanning capabilities. Further tests would incorporate this instrumentation as an alternative survey method and evaluate the outcome using similar parameters as Table 5. 
Even though the CRP produce comparable data to the TLS dataset, the image network could be further improved in order to provide sufficient coverage throughout the arch.

\begin{tabular}{|c|c|c|c|}
\hline Factors to consider & $\begin{array}{c}\text { Prism based } \\
\text { monitoring }\end{array}$ & TLS & CRP \\
\hline $\begin{array}{c}\text { Relatively Accurate } \\
\text { enough? }\end{array}$ & High & Medium & Medium \\
\hline Requires targets? & Yes & No & No \\
\hline Latency & High & Medium & Low \\
\hline $\begin{array}{c}\text { Produce displacement } \\
\text { surface map? }\end{array}$ & No & Yes & Yes \\
\hline $\begin{array}{c}\text { Produce sections from } \\
\text { data? }\end{array}$ & No & Yes & Continuous \\
\hline Coverage & Medium & Continuous & Low \\
\hline Install Period & Medium & Medium & High \\
\hline $\begin{array}{c}\text { Calibration } \\
\text { Flexibility in }\end{array}$ & Medium & High & TLS to CRP \\
\hline $\begin{array}{c}\text { instrument location } \\
\text { Global registration } \\
\text { error }\end{array}$ & TS to TLS & TLS to TLS & $\sim 3 \mathrm{~mm}$ \\
\hline $\begin{array}{c}\text { Local feature } \\
\text { comparison }\end{array}$ & $\sim 1 \mathrm{~mm}$ & $\sim 7 \mathrm{~mm}$ & $2.6 \mathrm{~mm}$ \\
\hline $\begin{array}{c}\text { Typical profile } \\
\text { discrepancy }\end{array}$ & n/a & $2.5 \mathrm{~mm}$ & $\begin{array}{c}\text { 2mm } \\
\text { (max 6.8mm) }\end{array}$ \\
\hline
\end{tabular}

Table 5 - Comparison of capabilities of different instrumentation for monitoring

\section{Acknowledgements}

This research is based on a collaborative project funded by the EPSRC (Engineering and Physical Sciences Research Council) and Network Rail through the VEIV (Virtual Environments Imaging \& Visualisation) Engineering Doctorate Centre at University College London. The authors would like to thank the Survey team at Costain working on the London Bridge Redevelopment Project, in particular Dean Bain and Rob Williams, who organised access to the site and equipment as well as providing continuous support towards the study. 


\section{References:}

Abellán, A., M. Jaboyedoff, et al. (2009). "Detection of millimetric deformation using a terrestrial laser scanner: experiment and application to a rockfall event." Natural Hazards and Earth System Sciences 9(2): 365-372.

Abellán, A., J. Vilaplana, et al. (2011). "Rockfall monitoring by Terrestrial Laser Scanning-case study of the basaltic rock face at Castellfollit de la Roca (Catalonia, Spain)." Natural Hazards and Earth System Science 11(3): 829-841.

Alba, M., L. Fregonese, et al. (2006). Structural monitoring of a large dam by terrestrial laser scanning. Proceedings of of the ISPRS Commission V Symposium, Dresden.

Alba, M. and M. Scaioni (2010). Automatic detection of changes and deformation in rock faces by terrestrial laser scanning. Proceedings of the ISPRS Commission V Mid-Term Symposium 'Close Range Image Measurement Techniques.

Allan, A. L. (2007). Principles of geospatial surveying, Whittles Pub.

Baltsavias, E. P. (1991). Multiphoto geometrically constrained matching, ETH Zurich, Switzerland.

Besl, P. J. and N. D. McKay (1992). "A method for registration of 3-D shapes." IEEE Transactions on pattern analysis and machine intelligence 14(2): 239-256.

Chow, J., D. Lichti, et al. (2012). "Accuracy assessment of the faro focus3D and leica HDS6100 panoramic type terrestrial laser scanner through point-based and plane-based user self-calibration." Proceedings of the FIG Working Week: Knowing to Manage the Territory, Protect the Environment, Evaluate the Cultural Heritage, Rome, Italy: 6-10.

Erdélyi, J., Kopáčik, A., Ilkovičová, L, Lipták, I. and Kajánek, P (2014). Deformation Monitoring of a Parabolic Roof Structure using TLS. INGEO 2014 - 6th International Conference on Engineering Surveying. Prague, Czech Republic.

FARO. (2013). "FARO Focus3D: Features, Benefits \& Technical Specifications." Retrieved 13 January 2014, from http://www.faro.com/products/3d-surveying/laserscanner-faro-focus-3d/overview.

FARO. (2014). "Faro Laser Scanners X Series." Retrieved 15 September, 2014, from http://www.faro.com/en-us/products/3d-surveying/faro-focus3d/overview.

Fryer, J., R. Parberry, et al. (1992). "Analysis of as-built cylindrical shapes." Australian Journal of Geodesy, Photogrammetry and Surveying 56: 91-109.

Gordon, S. J., D. Lichti, et al. (2005). Structural deformation measurement using terrestrial laser scanners, Curtin University of Technology.

Granshaw, S. (1980). "Bundle adjustment methods in engineering photogrammetry." The Photogrammetric Record 10(56): 181-207.

James, M. and S. Robson (2012). "Straightforward reconstruction of 3D surfaces and topography with a camera: accuracy and geoscience application." Journal of Geophysical Research: Earth Surface (2003-2012) 117(F3). 
Jiang, R., D. V. Jáuregui, et al. (2008). "Close-range photogrammetry applications in bridge measurement: literature review." Measurement 41(8): 823-834.

Kang, Z., L. Tuo, et al. (2012). "Continuously deformation monitoring of subway tunnel based on terrestrial point clouds." Int. Arch. Photogramm. Remote Sens. Spat. Inf. Sci 39: 199-203.

Kopáčik, A., Erdélyi, J., Lipták, I. and Kyrinovič, P (2013). Deformation of Bridge Structures using TLS. 2nd Joint International Syposium in Deformation Monitoring, Univeristy of Nottingham.

Laefer, D. F., L. Truong-Hong, et al. (2014). "Crack detection limits in unit based masonry with terrestrial laser scanning." NDT \& E International 62: 66-76.

Leica Geosystems, A. (2010). "Leica Viva TS15." Data sheet, Herbrugg.

Lerma García, J. L., B. Van Genechten, et al. (2008). 3D Risk Mapping. Theory and Practice on Terrestrial Laser Scanning. Training Material Based on Practical Applications, Universidad Politécnica de Valencia, Valencia.

Lindenbergh, R. and N. Pfeifer (2005). "A statistical deformation analysis of two epochs of terrestrial laser data of a lock." Proc. of Optical 3D Measurement Techniques 2: 61-70.

Luhmann, T., S. Robson, et al. (2013). Close-range Photogrammetry and 3D Imaging, De Gruyter.

Monserrat, O. and M. Crosetto (2008). "Deformation measurement using terrestrial laser scanning data and least squares 3D surface matching." ISPRS Journal of Photogrammetry and Remote Sensing 63(1): 142-154.

Nuttens, T., A. De Wulf, et al. (2010). High Resolution Terrestrial Laser Scanning for Tunnel Deformation Measurements. FIG Congress 2010: Facing the Challenges - Building the Capacity, Sydney, Australia.

Nuttens, T., A. De Wulf, et al. (2012). Application of laser scanning for deformation measurements: a comparison between different types of scanning instruments. Proceedings of the FIG Working Week.

Nuttens, T., C. Stal, et al. (2014). "Methodology for the ovalization monitoring of newly built circular train tunnels based on laser scanning: Liefkenshoek Rail Link (Belgium)." Automation in Construction 43: 1-9.

Olsen, M. J., F. Kuester, et al. (2009). "Terrestrial laser scanning-based structural damage assessment." Journal of Computing in Civil Engineering 24(3): 264-272.

Park, H., H. Lee, et al. (2007). "A new approach for health monitoring of structures: terrestrial laser scanning." Computer-Aided Civil and Infrastructure Engineering 22(1): 19-30.

Puente, I., H. González-Jorge, et al. (2012). "Deformation Monitoring of Motorway Underpasses Using Laser Scanning Data." ISPRS-International Archives of the Photogrammetry, Remote Sensing and Spatial Information Sciences 1: 235-238.

Reshetyuk, Y. (2010). "Direct georeferencing with GPS in terrestrial laser scanning." Z f V-Zeitschrift für Geodäsie, Geoinformation und Landmanagement 135(3): 151-159.

Riegl. (2014). "Riegl Laser Measurement Systems." Retrieved 15th September, 2014, from http://www.riegl.com/nc/products/terrestrial-scanning/produktdetail/product/scanner/33/. 
Riveiro, B., H. González-Jorge, et al. (2013). "Validation of terrestrial laser scanning and photogrammetry techniques for the measurement of vertical underclearance and beam geometry in structural inspection of bridges." Measurement 46(1): 784-794.

Riveiro, B., P. Morer, et al. (2011). "Terrestrial laser scanning and limit analysis of masonry arch bridges." Construction and building materials 25(4): 1726-1735.

Schneider, D. (2006). Terrestrial laser scanning for area based deformation analysis of towers and water dams. Proc. of 3rd IAG/12th FIG Symp., Baden, Austria, May.

Schuhmacher, S. and J. Böhm (2005). "Georeferencing of terrestrial laserscanner data for applications in architectural modeling."

Uren, J. and W. F. Price (2010). Surveying for engineers, Palgrave Macmillan.

Vezočnik, R., T. Ambrožič, et al. (2009). "Use of terrestrial laser scanning technology for long term high precision deformation monitoring." Sensors 9(12): 9873-9895.

Werner, T. and D. Morris (2010). 3D Laser Scanning for Masonry Arch Bridges. FIG Congress 2010: Facing the Challenges - Building the Capacity, Sydney, Australia.

Westoby, M., J. Brasington, et al. (2012). "'Structure-from-Motion' photogrammetry: A low-cost, effective tool for geoscience applications." Geomorphology 179: 300-314.

Yoon, J.-S., M. Sagong, et al. (2009). "Feature extraction of a concrete tunnel liner from 3D laser scanning data." NDT \& E International 42(2): 97-105. 